Ana Carolina Brandão de Campos Fonseca PINTO ${ }^{1}$ Masao IWASAKI ${ }^{1}$ Cláudia FIGUEIREDO' ${ }^{1}$

Sílvia Renata Gaido CORTOPASSI ${ }^{1}$

Franklin de Almeida STERMAN ${ }^{1}$

Correspondência para: ANA CAROLINA BRANDÃO DE CAMPOSFONSECA PINTO

Departamento de Cirurgia da Faculdade Medicina Veterinária e Zootecnia Universidade de São Paulo

Av. Prof. Dr. Orlando Marques de Paiva, 87, Cidade Universitária 05508-270 - São Paulo - SP anacarol@usp.br

Recebido para publicação: 24/03/2004 Aprovado para publicação: 24/03/2004

\title{
Tomografia computadorizada do tórax de cadelas portadoras de neoplasias mamárias malignas. I - determinação da técnica do exame
}

\author{
1 - Departamento de Cirurgia da Faculdade de Medicina Veterinária e \\ Zootecnia da Universidade de São Paulo, São Paulo - SP
}

\section{Resumo}

Visto a importância das neoplasias mamárias malignas na clínica médico-veterinária e as novas perspectivas do diagnóstico por imagem na avaliação de pacientes portadores dessas neoplasias, o presente trabalho visou analisar alguns aspectos técnicos relativos ao exame de tomografia computadorizada contrastada da cavidade torácica quais sejam, tempo de realização do exame, escolha da espessura dos cortes transversais, qualidade de contrastação dos vasos mediastinais, abertura de janela e nível para obtenção das imagens de pulmão, mediastino e arcabouço ósseo, tendo em vista a escassez de informações na literatura consultada. Para tanto, foram realizados exames de tomografia computadorizada contrastada da cavidade torácica de vinte fêmeas da espécie canina, de diferentes raças e idades, portadoras de neoplasias mamárias malignas encaminhadas ao Serviço de Diagnóstico por Imagem do Hospital Veterinário da Faculdade de Medicina Veterinária e Zootecnia da Universidade de São Paulo pelos Serviços de Obstetrícia e Ginecologia e de Cirurgia de Pequenos Animais da mesma instituição. Concluiu-se que, o tempo médio para realização da tomografia contrastada completa do tórax, com aproximadamente trinta cortes foi de 30 minutos; o posicionamento em decúbito esternal com os membros tracionados cranialmente, e a administração de contraste iodado hidrossolúvel por via intravenosa no volume aproximado de $2 \mathrm{ml} / \mathrm{kg}$ de peso vivo, sendo dois terços da dose administrados em bolo e o complemento sob infusão contínua mostraram-se adequados para realização do exame tomográfico contrastado do tórax; a escolha da espessura dos cortes de 10 milímetros para animais com mais de trinta quilos e de 5 milímetros para animais com menos de trinta quilos mostrou-se adequada para avaliação de todo o tórax buscando-se atingir uma média de trinta cortes; as seleções de janela e nível para aquisição das imagens pulmonares, mediastinais e de arcabouço ósseo apresentaram-se adequadas à avaliação a partir dos valores de abertura de janela de: 1500 unidades Hounsfield (UH) com um nível entre -550 e -650 UH para pulmão, entre 250 e $300 \mathrm{UH}$ com um nível entre 0 e $50 \mathrm{UH}$ para mediastino e para avaliação do arcabouço ósseo de 1500 UH com um nível entre 50 e 350 UH.

\section{Introdução}

Tomografia é uma imagem seccional, formada livre de sobreposição de estruturas adjacentes e que pode ser produzida em planos transversal, dorsal, sagital e oblíquo.

\author{
Palavras-chave: \\ Tomografia \\ computadorizada. \\ Técnica. \\ Cavidade torácica. \\ Cadelas.
}

Todas as técnicas de imagem que gerem cortes seccionais como a ultra-sonografia, a tomografia computadorizada e a ressonância magnética, por exemplo, são formas de tomografia ${ }^{1}$. A tomografia computadorizada (TC) é, portanto, um método de 
1 GENERAL ELETRIC

2 IBF-INDÚSTRIA BRASILEIRA DE FILMES

3 Kodak Brasileira Com. Ind. LTDA

4EASTMAN KODAK COMPANY

1 Acepran ${ }^{\circledR}$ a 0,2\% - Univet S.A.

2 Dolantina - Cristália, São Paulo, SP

3 Diprivan - Zeneca, São Paulo, SP

4 Samurai III - Takaoka, São Paulo, SP diagnóstico por imagem que produz cortes seccionais de uma região do corpo através de raios X e computadores, com a vantagem sobre a radiografia convencional de apresentar grande sensibilidade em diferenciar pequenas atenuações dos raios $\mathrm{X}$ e ser isenta de sobreposição de estruturas ${ }^{2,3,4}$. Aspectos técnicos relacionados ao procedimento do exame tomográfico como: seleções de quilovoltagem, miliamperagem e tempo de aquisição das imagens ${ }^{5}$, contenção química do paciente ${ }^{6}$, escolha do decúbito para realização do exame tomográfico ${ }^{6,7,8,9,10}$, seleção da espessura dos cortes transversais 9,10 , uso de contraste, sugestão de valores de abertura de janela e de nível para obtenção de imagens de boa qualidade para interpretação em medicina veterinária 3,6,11 têm sido apresentados pelos autores.

A utilização do exame tomográfico no estudo da cavidade torácica permite avaliação de campos pulmonares, mediastino, pleuras e espaço pleural e parede torácica ${ }^{1,6,11,12,13,14}$. De Haan, Papageorges e Kraft ${ }^{12}$ indicam a utilização do exame tomográfico na clínica médica veterinária para: detecção de linfoadenopatias hilares ou mediastinais, pesquisa de nódulos pequenos e múltiplos, no direcionamento da aspiração com agulha fina de um nódulo quando a fluoroscopia não estiver disponível e para detecção precoce e mensuração de metástases pulmonares.

Na medicina, a TC é considerada a modalidade de imagem mais sensível para a detecção de metástases pulmonares ${ }^{3,12,15}$. Todavia, para utilização em animais, ela só está disponível em grandes centros de pesquisa tendo em vista os custos para aquisição e manutenção do equipamento. É o exame radiográfico indicado como meio de eleição para a investigação inicial da cavidade torácica dos animais ${ }^{3,17,18}$ e complemento fundamental ao exame físico em cadelas com tumor de glândulas mamárias ${ }^{17,18,19,20}$.

As neoplasias mamárias estão entre as neoplasias que mais freqüentemente acometem as fêmeas da espécie canina ${ }^{16,24}$, para Brodey, goldsclimidt e Rosze ${ }^{17}$ cerca de $52 \%$ e $50 \%$ segundo Keef ${ }^{22}$. Fildler, Adt e Brodey ${ }^{23}$ sugerem que aproxi-madamente $50 \%$ dessas neoplasias são malignas. Para Brodey, Golds-Chmidt e Roszel ${ }^{17}$ o câncer da glândula mamária é a causa mais comum de metástases pulmonares em cadelas.

Tendo em vista a relevância das neoplasias mamárias malignas na clínica médico-veterinária e as novas perspectivas do diagnóstico por imagem na avaliação de pacientes portadores dessas neoplasias e, tendo em vista a escassez de informações na literatura consultada, no presente trabalho, visou-se a análise de aspectos técnicos relativos ao exame de tomografia computadorizada contrastada da cavidade torácica quais sejam, tempo de realização do exame, espessura dos cortes transversais, contrastação dos vasos mediastinais, abertura de janela e nível para obtenção das imagens de pulmão, mediastino e arcabouço ósseo.

\section{Materiais e Métodos}

Foram realizados exames de tomografia computadorizada contrastada da cavidade torácica de vinte fêmeas da espécie canina, de diferentes raças e idades, portadoras de neoplasias mamárias malignas atendidas pelos Serviços de Obstetrícia e Ginecologia e de Cirurgia de Pequenos Animais do Hospital Veterinário da Faculdade de Medicina Veterinária e Zootecnia da Universidade de São Paulo e, encaminhadas ao Serviço de Diagnóstico por Imagem do Hospital Veterinário da Faculdade de Medicina Veterinária e Zootecnia da Universidade de São Paulo para realização do exame tomográfico em equipamento da marca GE modelo CTMAX $640^{1}$ de terceira geração. As imagens foram fotografadas em câmera multiformato MFC640 ${ }^{1}$ em filmes de marcas MN NIF Agfa IBF Medix ${ }^{2}$ e Kodak Ektanscan $\mathrm{M}^{3}$ tamanho $35 \times 43 \mathrm{~cm}$, os quais foram revelados e fixados em Processadora Automática RPX-OMAT Processor ${ }^{4}$.

Os vinte animais selecionados neste 
estudo foram submetidos à anestesia geral para possibilitar os posicionamentos desejados, bem como, a imobilidade necessária para a realização da seqüência de cortes tomográficos. Para tanto, os animais foram pré-tratados com 0,05 a $0,1 \mathrm{mg} / \mathrm{kg}$ de acepromazina ${ }^{1}$ e 3,0 a 5,0 mg/ $\mathrm{kg}$ de meperidina ${ }^{2}$ através da via intramuscular. Decorridos 20 minutos, a veia cefálica foi cateterizada e a indução da anestesia foi realizada através da administração de propofol $^{3}$ na dose de $5,0 \mathrm{mg} / \mathrm{kg}$. Uma vez que os animais apresentaram relaxamento da região mandibular e ausência do reflexo laringotraqueal, realizou-se a intubação endotraqueal com sonda de diâmetro adequado a qual foi conectada ao circuito circular do aparelho de anestesia ${ }^{4}$. Os animais receberam isofluorano ${ }^{5}$ em oxigênio a 100\% em concentração adequada para permitir a manutenção do $2^{\circ} / 3^{\circ}$ plano de anestesia. Durante todo o procedimento, realizou-se a monitoração da freqüência e ritmo cardíaco $^{6}$, freqüência respiratória e pressão arterial sistêmica não invasiva ${ }^{6}$. Os pacientes foram então posicionados em decúbito esternal com os membros torácicos tracionados cranialmente, evitando a superposição com a cavidade torácica. Os cortes tranversais se iniciaram na entrada do tórax e se estenderam até a porção mais caudal dos lobos pulmonares caudais.

$\mathrm{O}$ ajuste de técnica no aparelho de tomografia foi de $120 \mathrm{kVp}$ e 22 a $77 \mathrm{~mA}$, com 3 a 4,8 segundos de tempo de aquisição. Assim, dentre as possibilidades de calibrações oferecidas pelo aparelho, que variam de 22 a 110 em relação a miliamperagem e de 3 a 4,8 segundos em relação ao tempo, optou-se por manter uma miliamperagem de 55 com 3 segundos de tempo em 16 dos animais. Em três pacientes, independentemente do porte, utilizou-se $22 \mathrm{~mA}$ com 3 segundos de tempo e, apenas em um, o exame foi realizado com $77 \mathrm{~mA}$ e 5 segundos de tempo permanecendo em todos os exames o pico da quilovoltagem constante de $120 \mathrm{kVp}$.

A espessura dos cortes foi de 5 ou os cortes, dependendo do porte do paciente, buscando-se atingir um número médio de 30 cortes. Para tanto em alguns pacientes foram realizados cortes adicionais de 2 ou $5 \mathrm{~mm}$. As imagens foram obtidas após injeção intravenosa de contraste iodado hidrossolúvel não iônico Omnipaque 300?7 no volume aproximado de $2 \mathrm{ml} / \mathrm{kg}$ de peso, sendo dois terços da dose administrados em bolo e o complemento sob infusão contínua (gotejamento sem ocorrência de fio contínuo). Em um dos animais, com peso de $40 \mathrm{~kg}$, utilizou-se o contraste iodado hidrossolúvel iônico Hypaque ?8 $50 \%$. As imagens adquiridas foram fotografadas em câmera multiformato MFC $640^{9}$, com seleções de janela e nível que permitissem adequada avaliação de campos pulmonares, mediastino e arcabouço ósseo. Estas seleções de janela e nível partiram de adequações dos valores propostos por Burk $^{11}$, Stickle; Hathcock ${ }^{6}$ e Schwarz; Tidwell ${ }^{18}$.

Foram então registrados os aspectos técnicos relativos ao procedimento, tais sejam: tempo de realização do exame, qualidade de contrastação dos vasos mediastinais (ótima, suficiente e regular), abertura de janela e nível para obtenção das imagens que permitissem adequada avaliação de pulmões, mediastino e arcabouço ósseo, para posterior descrição.

\section{Resultados}

Os animais que participaram desta pesquisa, apresentaram características corpóreas bastante distintas, assim a distribuição dos animais estudados nesta pesquisa de acordo com a raça foi de: cinco animais da raça Dachushund (25\%), três $(15 \%)$ da raça Cocker, dois (10\%) Poodles, um $(5 \%)$ da raça Terrier brasileiro (Fox Paulistinha), um (5\%) Pinscher, um (5\%) Mastin Napolitano, um (5\%) da raça Pastor Alemão e $6(30 \%)$ sem definição racial (SRD). As idades destas cadelas variaram de quatro a catorze anos, sendo que 50\% dos animais tinham idades entre 10 e 14 anos, 
$35 \%$ variaram entre 7 e 9 anos, e apenas $15 \%$ tinham idades entre 4 e 6 anos. Quanto ao peso, observou-se uma variação entre 4,5 e $45 \mathrm{~kg}$, com $55 \%$ dos animais pesando até $10,9 \mathrm{~kg}, 20 \%$ pesando entre 11 e $20,9 \mathrm{~kg}, 15 \%$ das cadelas pesando entre 21 e $30,9 \mathrm{~kg}$ e apenas dois animais pesando mais de $31 \mathrm{~kg}$ $(10 \%)$. Apesar desta heterogeneidade do grupo em estudo, os diferentes ajustes técnicos de $120 \mathrm{kVp}$ com $22 \mathrm{~mA}$ e $3 \mathrm{~s}$, $120 \mathrm{kVp}$ com $55 \mathrm{~mA}$ e $3 \mathrm{~s}$ ou $120 \mathrm{kVp}$ com 77 com 3 ou 4,8s, não proporcionaram diferenças visuais importantes nos exames tomográficos, como a presença de ruídos, que comprometessem a interpretação das imagens.

O tempo médio para realização dos exames tomográficos, considerando-se a radiografia digital como tempo zero e o último corte como final do exame, foi de 29 minutos e 39 segundos, sendo que o exame mais breve durou 21 minutos e o mais longo 51 minutos. Vale ressaltar que em alguns exames, condições técnicas de aquecimento do aparelho ou de movimentação do paciente (onde houve necessidade de ser realizada uma nova radiografia digital para se realizar cortes adicionais finalizando o exame) implicaram em aumento do tempo de realização do exame de forma não quantificada neste estudo.

As janelas e os níveis escolhidos para avaliação de pulmão, mediastino e arcabouço ósseo mantiveram-se constantes do início ao final do exame em $70 \%$ dos casos e necessitaram de ajustes 30\% dos casos (para janela de mediastino e nível de mediastino e osso) em diferentes cortes de um mesmo exame.

Para 95\% dos animais a abertura das janelas para pulmão foi de $1500(\mathrm{HU})$, para apenas um animal selecionou-se abertura da janela de 2000; os níveis selecionados para pulmão variaram de -350 a $-800(\mathrm{HU})$, sendo que em $65 \%$ dos casos o nível variou de -550 a -650. A abertura da janela para mediastino variou de 250 a $400(\mathrm{HU})$, sendo que em $90 \%$ dos exames a abertura da janela para mediastino variou de 250 a 300. O nível para mediastino variou de -37 a $80(\mathrm{HU})$, sendo que em $80 \%$ dos exames o nível variou de 0 a 50. Para o arcabouço ósseo a abertura da janela manteve-se constante em 1500 (HU) em dezenove dos vinte exames (95\%) e em 5\% foi usada abertura de 2000. Com relação ao nível este variou de 30 a 480 (HU) sendo que em $40 \%$ dos exames o nível variou de 50 a 150 , e em $30 \%$ variou de 250 a 350 (HU).

A espessura dos cortes selecionada em $75 \%$ dos exames foi de 5 milímetros, sendo que, esses pacientes apresentavam menos de $30 \mathrm{~kg}$ de peso. Apenas em uma cadela com menos de $30 \mathrm{~kg}$ foi selecionada a espessura de 10 milímetros sendo que nesta, obteve-se apenas 22 cortes. Os demais 20\% dos exames foram realizados com 10 milímetros de espessura em animais com mais de trinta quilogramas. A espessura e o incremento dos cortes realizados mantiveram-se constantes em $90 \%$ dos exames (18 exames) e cortes adicionais com espessura diferente da primeira foram realizados em $10 \%$ dos casos (2 exames) a fim de se complementar o exame.

Em 10\% dos exames (dois animais) houve movimentação do paciente e foi realizada uma nova radiografia digital para se estabelecer os cortes finais a fim de completar o exame.

A qualidade de contrastação das estruturas vasculares do mediastino, obtida com a injeção de contraste iodado hidrossolúvel (não iônico ou iônico), foi classificada segundo a capacidade de individualização das estruturas vasculares em ótima, suficiente e regular; e se verificou a graduação ótima em 50\% dos exames, suficiente em $40 \%$ e regular em 10\%.

Em 50\% dos exames foram observados artefatos de moção nas imagens adquiridas.

\section{Discussão}

As diferentes técnicas empregadas neste estudo não interferiram na obtenção de imagens tomográficas com qualidade que possibilitasse a avaliação da cavidade torácica. Jones; Wilson; Bartels ${ }^{5}$ exemplificam as 
diferenças promovidas por diversos ajustes de técnica e espessura de cortes realizados em uma mesma região que não foi o escopo desta pesquisa. Todavia, visto que quanto menor a miliamperagem menor será o aquecimento do tubo, ou seja, podem se formar ruídos que degradam a qualidade da imagem ${ }^{5}$, acredita-se que o ajuste utilizado na maior parte dos exames com valor intermediário de miliamperagem $(120 \mathrm{kVp}$ com $55 \mathrm{~mA}$ e $3 \mathrm{~s})$ seria o mais apropriado.

Foi possível estabelecer que o tempo médio de duração do exame completo do tórax, com aproximadamente trinta cortes, realizado sob as condições estabelecidas (aparelho CT MAX 640 com 3 segundos de duração cada corte) é de cerca de trinta minutos, o que torna viável a realização do exame no momento pré-operatório diminuindo assim o número de procedimentos anestésicos. No entanto, cada caso deve ser analisado individualmente tendo em vista que algumas cirurgias podem necessitar de tempo operatório bastante longo e contra-indicar a associação do exame no mesmo tempo anestésico.

As seleções de janelas e níveis para obtenção das imagens que permitissem adequada avaliação de campos pulmonares, mediastino e arcabouço ósseo partiram de valores propostos por Burk ${ }^{11}$, Schwarz e Tidwell ${ }^{3}$ e Stickle e Hathcock ${ }^{6}$, porém adaptados segundo escolha pessoal de acordo com o porte do paciente. De forma geral, indica-se para pulmão uma abertura de janela de 1500 UH e um nível entre -550 e $-650 \mathrm{UH}$, para mediastino uma abertura de janela variando entre 250 e $300 \mathrm{UH}$ com um nível entre 0 e $50 \mathrm{UH}$ e para avaliação do arcabouço ósseo uma abertura de janela de 1500 UH com um nível entre 50 e 350 UH diferentemente do proposto por Stickle e Hathoock ${ }^{6}$ e semelhante aos valores indicados por Schwarz e Tidwell ${ }^{3}$. A partir dos valores propostos, deve-se selecionar os valores de janela e nível, de acordo com a preferência pessoal, e em conformidade com o que se pretende estudar (pulmão, mediastino e arcabouço ósseo).
O posicionamento em decúbito esternal com os membros tracionados cranialmente segundo proposição de Ahlberg et $\mathrm{al}^{7}$, Burk ${ }^{11}$ e Stickle e Hathcock ${ }^{6}$, mostrouse de fácil execução desde que a formação na mama não tenha dimensões muito grandes. Ainda, no que diz respeito ao decúbito, devido à observação de quadros pulmonares alveolares relacionados com atelectasia e estase, e tendo em vista que os nossos pacientes têm que estar anestesiados por cerca de trinta minutos para a realização do exame, acreditamos que devam ser realizados novos estudos com um número significativo de animais para que se possa estabelecer e comparar os efeitos dos posicionamentos em decúbito esternal e dorsal nas imagens tomográficas.

A escolha da espessura do corte entre 5 e $10 \mathrm{~mm}$ variou na dependência do porte do animal com a intenção de obter uma média de trinta cortes. Assim nos animais com peso acima de $30 \mathrm{~kg}$ foram realizados cortes com $10 \mathrm{~mm}$ obtendo-se um total de aproximadamente 30 cortes. Somente em um animal com menos de 30 quilos, pesando 12 quilos, empregou-se cortes com $10 \mathrm{~mm}$ de espessura, obtendo-se um total de apenas 22 cortes. Deste modo, sugere-se para realização de exame tomográfico de todo o tórax, que a escolha da espessura do corte seja relacionada ao porte como foi proposto por Stickle e Hathcock ${ }^{6}$ e ao peso. Deste modo, indica-se para animais com mais de 30 quilos a espessura de 10 milímetros e para animais com menos de 30 quilos de 5 milímetros.

A movimentação do paciente, causada simplesmente pela respiração, ou pela anestesia superficial, é uma situação adversa que acaba promovendo artefatos na imagem tomográfica. Tais artefatos devem ser levados em consideração quando da interpretação das imagens visando analisar o quanto podem estar prejudicando a avaliação do exame. Neste estudo, observaram-se efeitos da movimentação em $50 \%$ dos casos, sendo que, em apenas um deles, a avaliação do exame ficou prejudicada. 
A realização de pesquisas que visem a adequação das técnicas anestésicas a fim de minimizar os indesejados artefatos de moção torna-se um rico campo de estudos. Outro aspecto prejudicial é a movimentação do animal, que promove mudança de posição do paciente na mesa de exame, perdendo-se a referência do posicionamento dos cortes, sendo necessário, assim, realizar nova radiografia digital para nova orientação dos cortes transversais, prolongando o tempo do exame como ocorreu em especial com um animal que teve o tempo de duração do exame de 51 minutos. O prolongamento do tempo de duração do exame, neste estudo, também pode estar relacionado ao aquecimento do aparelho, que nesta situação interrompia a realização de outros cortes por tempo não quantificado neste trabalho.

A utilização de contraste iodado hidrossolúvel, para melhor distinção entre estruturas vasculares do mediastino e possíveis formações, como sugeriram Marincek e Young ${ }^{9}$ e Widmer ${ }^{10}$, foi realizada por via intravenosa no volume aproximado de $2 \mathrm{ml} / \mathrm{kg}$ de peso, sendo dois terços administrados em bolo e o complemento sob infusão contínua. Essa técnica mostrou-se adequada para realização do exame tomográfico contrastado do tórax obtendo classificação ótima ou suficiente para individualização das estruturas vasculares em $90 \%$ dos exames. A utilização do contraste iônico (Hypaque $^{\circledR 18} 50 \%$ ) em um dos pacientes que recebeu $80 \mathrm{ml}$ de contraste deveu-se às boas condições clínicas em que ela se apresentava, e ao alto custo do contraste não iônico para ser administrado em grandes volumes. Essa mudança na escolha do contraste, de não iônico para iônico, não permitiu que se inferisse se podem haver diferenças na qualidade de contrastação dos vasos com o uso de diferentes contrastes iodados, já que nesta pesquisa procurou-se utilizar a mesma dosagem de iodo.

\section{Conclusões}

Este estudo permitiu definir alguns aspectos técnicos que envolvem a realização do exame tomográfico contrastado do tórax de cadelas nas condições previstas nesta pesquisa. Assim foram analisados: o tempo médio para realização da tomografia contrastada completa do tórax, o posicionamento dos animais, a utilização de contraste iodado hidrossolúvel para individualização das estruturas vasculares do mediastino, a escolha da espessura dos cortes tomográficos, as seleções de janela e nível para aquisição das imagens pulmonares, mediastinais e de arcabouço ósseo. Nesta pesquisa foi possível demonstrar a viabilidade de realização do exame tomográfico do tórax realizado, sob as condições aqui apresentadas, tanto no que se refere ao estudo anatômico quanto a sua aplicação na investigação clínica. Frente ao exposto e, tendo em vista a escassez de informações pertinentes a aspectos básicos relativos a técnica para realização e análise do exame tomográfico do tórax em animais, cabe ressaltar que novos estudos devam ser realizados para que os achados tomográficos passem a ser interpretados com maior propriedade tais como: novas abordagens anestésicas que reduzam os artefatos de moção e possam permitir a realização do corte tomográfico no pico da inspiração e que definam as vantagens e desvantagens da realização do exame com o animal em decúbito esternal e dorsal.

\section{Computed tomography exam of the thorax of bitches with malignant mammary gland tumors. I - Technical exam determination}

\begin{abstract}
Given the importance of malignant mammary gland tumors in veterinary medicine clinics, new perspectives of diagnostic imaging in order to evaluate pacients that have this neoplasia, and the lack of information about it in literature, this research intended to analyse some technical aspects of the contrast computed tomography scanning. The scanning time, the choice of the axial slices thickness,
\end{abstract}

Key-words: Computed tomography. Technique. Thoracic cavity. Bitches. 
quality of the mediastinal vases contrast and the window width and level were studied in order to have images that make possible the evaluation of lungs, mediastinum, and bones. This research was performed at the Diagnostic Imaging Service of the Veterinary School Hospital of the Faculdade de Medicina Veterinária e Zootecnia at the University of São Paulo in twenty, different breed and age, bitches with malignant mammary gland tumors that were examined at the Obstetric and Ginecology and Small Animal Surgery Services of the same hospital. The average time for the complete contrast computed tomography scanning of the thorax, with nearly 30 slices, was thirty minutes; the ventral recumbency with the cranial traction of thoracic limbs, and the administration of intravenously iodine contrast medium $(2 \mathrm{ml} / \mathrm{kg})$ that was given; two thirds of the dose in bolus and the complement under continuing infusion presented to be appropriate for the thoracic contrast computed tomography scanning. The 10 milimeters thickness for animals weighting over 30 kilograms and 5 milimeters for animals weighting under 30 kilograms presented to be appropriate in order to reach an average of thirty slices; the window and level selections to obtain pulmonary, mediastinal and bone images presented appropriate to be evaluated starting from window width 1500 Hounsfield unit (HU) with level varyng from 550 e -650 for lungs, window width between 250 to $300 \mathrm{HU}$ with level varyng from 0 e 50 for mediastinum and window width of 1500 with the level between 50 and $350 \mathrm{HU}$.

\section{Referências}

1 SUTER, P. F.; et al. radiographic recognition of primary and metastatic pulmonary neoplasms of dogs and cats. Journal of American Veterinary Radiology Society, v. 15, n. 3, p. 3-25, 1974.

2 HATHCOCK, J. T.; STICKLE, R. L. Principles and concepts of computed tomography. Veterinary Clinics of North America. Small Animal Practice, v. 23, n. 2, p. 399-415, 1993.

3 SCHWARZ, L. A.; TIDWELL, A. S. Alternative imaging of the lung. Clinical Techniques in Small Animal Practice, v. 14, n. 4, p. 187-206, 1999.

4 TIDWELL, A. S. Diagnostic pulmonary imaging. Problems in Veterinary Medicine, v. 4, n. 2, p. 239264, 1992.

5 JONES, J C.; WILSON, M. E.; BARTELS, J. E. A Review of high resolution computed tomography and a proposed technique for regional examination of the canine lumbosacral spine. Veterinary Radiology and Ultrasound, v. 35, n. 5, p. 339-346, 1994.

6 STICKLE, R. L.; HATHCOCK, J. T. Interpretation of computed tomographic images. Veterinary Clinics of North America. Small Animal Practice, v. 23, n. 2, p. 417-435, 1993.

7 AHLBERG, N. E. A computed tomographic study of volume and $X$-ray attenuation of the lungs of beagles in various body positions. Veterinary Radiology, v. 26, n.
2, p. 43-47, 1985.

8 BURK, R. L. Radiographic examination of the cardiopulmonary system. Veterinary Clinics of North America. Small Animal Practice, v. 13, n. 2, p. 241258, May 1983.

9 MARINCEK, B.; YOUNG, S. Computed tomography of spontaneous canine neoplasms. Veterinary Radiology, v. 21, n. 4, p. 181-184, 1980.

10 WIDMER, W. R. Alternate imaging for the diagnosis of cancer. In: MORRISON, W. B. Cancer in dogs and cats: medical and surgical management. Baltimore: Williams \& Wilkins, 1998. cap. 16, p. 187-213.

11 BURK, R. L. Computed tomography of thoracic diseases in dogs. Journal of American Veterinary Medicine Association, v. 199, n. 5, p. 617-621, 1991.

12 DE HAAN, C. E.; PAPAGEORGES, M.; KRAFT, S. L. Radiographic diagnosis (pulmonary mass). Veterinary Radiology, v. 32, n. 2, p. 75-77, 1991.

13 FEENEY, D. A.; FLETCHER, T. F.; HAARDY, R. M. Atlas of correlative imaging anatomy of the normal dog ultrasound and computed tomography. Philadelphia: W. B. Saunders, 1991. p. 209-210, 335352.

14 JAKOVLJEVIC, S.; MORRISON, W. B. Using conventional radiography in cancer diagnosis and monitoring. In: MORRISON, W. B. Cancer in dogs and cats: medical and surgical management. Baltimore: Williams \& Wilkins, 1998. cap. 14, p. 147-165. 
15 CONCES, D. J.; et al. T1N0Mo Lung cancer: evaluation with CT. Radiology, v. 170, p. 643-646, 1989.

16 ROBERTS, R.; BANKS, W. C. Radiografhic considerations in the normal and altered cardiac silhouette of the canine patient. The Southwestern Veterinarian, v. 26, n. 1, p. 11-16, 1972.

17 BRODEY, R. S.; GOLDSCHMIDT, M. H.; ROSZEL, J. R. Canine mammary gland neoplasms. Journal of the American Animal Hospital Association, v. 19, p. 61-90, Jan./ Feb. 1983.

18 FERGUSON, H. R. Canine mammary gland tumors. Veterinary Clinics of North America. Small Animal Practice, v. 15, n. 3, p. 501-511, 1985

19 GOBELLO, C.; CORRADA, Y. Canine mammary tumors: an endocrine clinical approach. Compendium, v. 23 , n. 8 , p. 705-709, 2001

20 TIEMESSEN, I. Thoracic metastases of canine mammary gland tumors. A radiographic study. Veterinary Radiology, v. 30, n. 6, p. 249-252, 1989.

21 MISDORP, W.; et al. (Comp.). Histological classification of mammary tumors of the dog and cat. Washington, D.C.: Armed Forces Institute of Pathology, 1999. (WHO International Histological Classification of Tumors of Domestic Animals; 2nd series, v. 1).

$22 \mathrm{KEEF}, \mathrm{D}$. A. Tumors of the genital system and mammary glands. In: ETTINGER, S. J.; FELDMAN, E. C. Textbook of veterinary internal medicine. 4. ed. Philadelphia: W. B. Saunders, 1995. cap. 131, p. 16991704.

23 FILDLER, I. J.; ADT, D. A.; BRODEY, R. S. Biological behavior of canine mammary neoplasms. Journal of Veterinary Medical Association, v. 151, n. 10, p. 13111318, 1967.

24 SUTER, P. F. Thoracic radiography: a text atlas of thoracic diseases of the dog and cat. Switzerland: Peter F. Suter, 1984. cap. 1, p. 1-45; cap. 11, p. 518-682.

25 TIDWELL, A. S. Advanced imaging concepts: a pictorial glossary of CT and MRI technology. Clinical Techniques in Small Animal Practice, v. 14, n. 2, p. 65111, 1999. 